\title{
A CT-Free Intraoperative Planning and Navigation System for High Tibial Dome Osteotomy
}

\author{
Gongli Wang ${ }^{1}$, Guoyan Zheng ${ }^{1}$, Paul Alfred Grützner ${ }^{2}$, Jan von Recum ${ }^{2}$, and \\ Lutz-Peter Nolte ${ }^{1}$
}

\author{
${ }^{1}$ M.E. Müller Research Center for Orthopaedic Surgery, Institute for Surgical Technology \\ and Biomechanics, University of Bern, Switzerland \\ \{Gongli.Wang, Guoyan.Zheng, Lutz.Nolte\} @MEMcenter.unibe.ch \\ http://www.MEMcenter.unibe.ch \\ ${ }^{2}$ BG Trauma Center Ludwigshafen, University of Heidelberg, Germany \\ pa.gruetzner@urz.uni-heidelberg.de, recum@bgu-ludwigshafen.de
}

\begin{abstract}
High tibial dome osteotomy is a well accepted but technically demanding surgical procedure. Common complications include postoperative malalignment of either under- or over-correction, pin penetration of the tibial plateau, and damage to the tibial dorsal neurovascular structures. In order to address all these problems, we developed a CT-free intraoperative planning and navigation system based on SurgiGATE system (Praxim-Medivision, La Tronche, France). Following acquisition of fluoroscopic images and registration of anatomic landmarks, a patient specific coordinate system is established. The deformity is measured intraoperatively and the surgical procedure is planned interactively. The osteotomy and deformity correction are performed under navigational guidance. The system holds the promise to improve the accuracy, reliability, and safety of this surgical procedure.
\end{abstract}

\section{Introduction}

High tibial osteotomy is a proven treatment for uni-compartmental osteoarthritis of the knee and other proximal tibial deformities, particularly in young and active patients for whom total knee replacement is not advised [1]. Dome osteotomy introduced by Jackson [2] is a widely accepted technique particularly in European countries. With this technique, a series of closed pinholes equidistant from a rotational axis is drilled and connected each other by using a sharp osteotome. The deformity correction is accomplished as two separated cylindrical bone fragments slide on each other around the central rotational axis (Fig. 1). The advantage of this technique is to correct severe deformities without loss of metaphyseal bone or leg length. Additionally, it provides large bone-to-bone contact area thus enhances the postoperative stability and hastens the bone union. These advantages are not available with other techniques such as opening or closing wedge osteotomies [3], [4], [5].

However, dome osteotomy is generally recognized as technically demanding with a long learning curve. Conventional preoperative planning and surgical techniques have so far been inaccurate, and often resulted in postoperative malalignment of either under- or over-correction, which is the main reason of early failure or poor long-term 
results [6]. With conventional techniques, it is difficult to achieve the clinical requirement of realigning the mechanical axis of the affected limb to within a narrow range of $\pm 2^{\circ}$ [7], [8]. An evaluation of recently reported clinical results (e.g., [9], [10], [11]) reveals that only $60 \%-80 \%$ of postoperative axial alignments lie within this critical region even when operation is performed with jig systems. Moreover, an inaccurate drilling or bone cutting with conventional techniques may cause pin penetration of the tibial plateau, intercondylar fracture, or damage to the tibial dorsal neurovascular structures. They are also common complications of this surgical procedure [6].

Computer assisted surgery technologies can address these problems. As one of the main contributions of previous works, CT-based intraoperative guidance systems [12] and robot-assisted systems [13] have been developed for closing wedge osteotomy. However, despite the advantage of accurate removal a wedge from the bone, these systems have limitations of extra radiation exposure and the potential risk of infection caused by implanted fiducial markers. A more recently introduced CT-free hybrid computer-assisted navigation system [14] provides a reliable solution for opening wedge osteotomy, which uses fluoroscopic images in place of the CT scan, since they are already routinely used in operating rooms. However, to the authors' best knowledge, there has been to date no publication exploring a computer-assisted system for high tibial dome osteotomy.

In this paper, an intraoperative planning and navigation system is proposed for tibial dome osteotomy, which aims to address all the common intraoperative technical problems and to make this surgical procedure more accurate, safe, and reproducible. The system provides following advantages: (1) accurate measurement of the deformity, (2) interactive planning of the surgical procedure, (3) precise performance of the deformity correction under navigational guidance, (4) avoidance of the osteotomy failures such as intraarticular pin penetration or damage to the tibial dorsal neurovascular structures, (5) creation of a congruous cylindrical osteotomy surface that offers maximal postoperative bony contact, thus encouraging rapid bony union.

\section{Materials and Methods}

\subsection{System Components}

The system (Fig. 2) is developed based on SurgiGATE system (Praxim-Medivision, La Tronche, France). An optoelectronic infrared tracking localizer (OptoTrack 3020, Northern Digital Inc., Waterloo, Ontario, Canada), mounted on a movable stand, is used to track the position of optical targets equipped with infrared light-emitting diodes. These targets are attached to anatomical bodies, the image intensifier of the $\mathrm{C}$ arm, and to other relevant surgical instruments.

A Sun ULTRA 10 workstation (Sun Microsystems Inc., Mountain View, Canada) is chosen for the image processing and visualization tasks. It is connected to the video output of the $\mathrm{C}$-arm for acquisition of fluoroscopic images. The workstation communicates with the infrared tracking system through customized software using client/server architecture. 


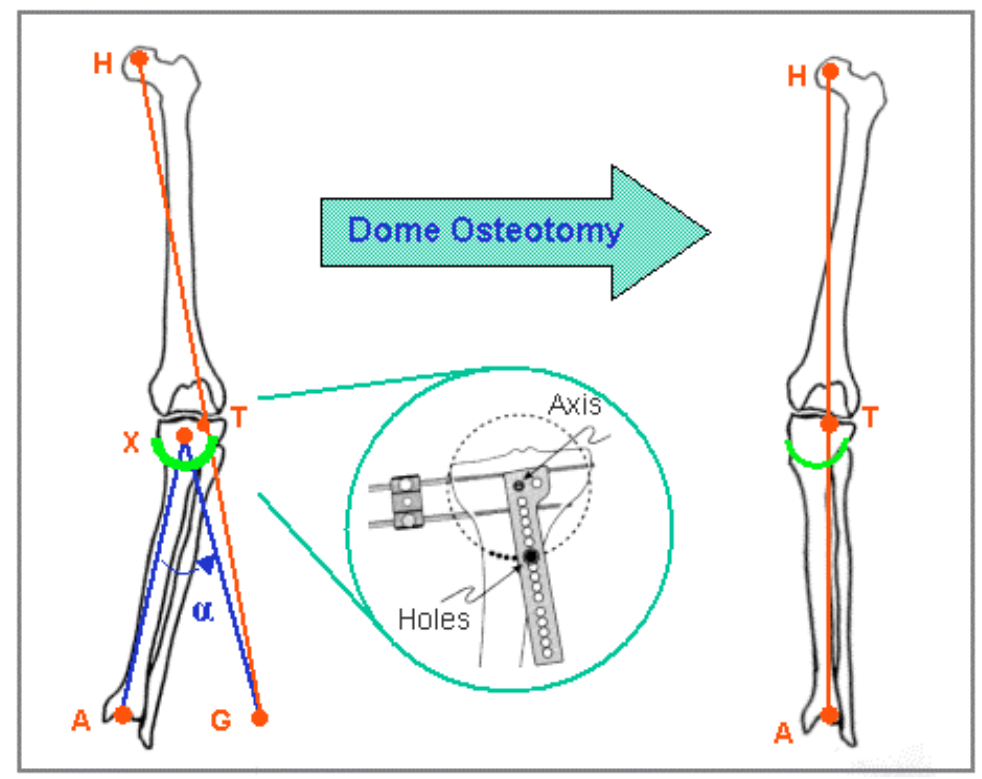

Fig. 1. Schematic of high tibial focal dome osteotomy. The goal of this surgical procedure is to realign the mechanical axis of the affected limb from the diseased compartment to the more healthy side. To accomplish this, a series of pinholes equidistant from a central rotational axis is drilled along anterior posterior direction with the help of a guiding tool (see close-up). The proximal and distal bone fragments are separated completely by connecting these pinholes each other with a sharp osteotome. The deformity is corrected as two cylindrical shaped bone fragments slide on each other rotating around the central axis under manual stress [3]. As shown in the image, $\mathrm{H}$ is the hip center, $\mathrm{A}$ is the ankle center, $\mathrm{X}$ is the rotational axis for deformity correction, $\mathrm{T}$ is the anticipated postoperative position of the weight bearing axis at the tibial plateau, $G$ is the corresponding postoperative ankle center, and $\alpha$ is the necessary rotational angle to correct the deformity

The navigated instruments can be divided into three groups according to their functionalities. The first group includes a calibrated fluoroscopic C-arm, a tool for measuring the gravitational direction, an accuracy checker for the verification of system accuracy, and a pointing device (pointer) for percutaneous digitization and landmarks verification. This group of instruments is used for image acquisition and landmark registration. The next group consists of a navigated drill and a chisel. They are used for cutting the bone. The last group includes three dynamic reference bases (DRBs), which are affixed to the operated limb for tracking the motion of the corresponding anatomies during operation. All surgical instruments and DRBs can be gas or steam sterilized.

\subsection{Landmark Registration and Deformity Measurement}

Intraoperatively, after the surgeon affixes DRBs at the femur and the tibia of the affected limb, the fluoroscopic images (Fig. 3) are acquired and uploaded into 
navigation system at the beginning of operation, including knee AP (anteriorposterior), knee lateral, hip AP, hip lateral, ankle AP, and ankle lateral images.

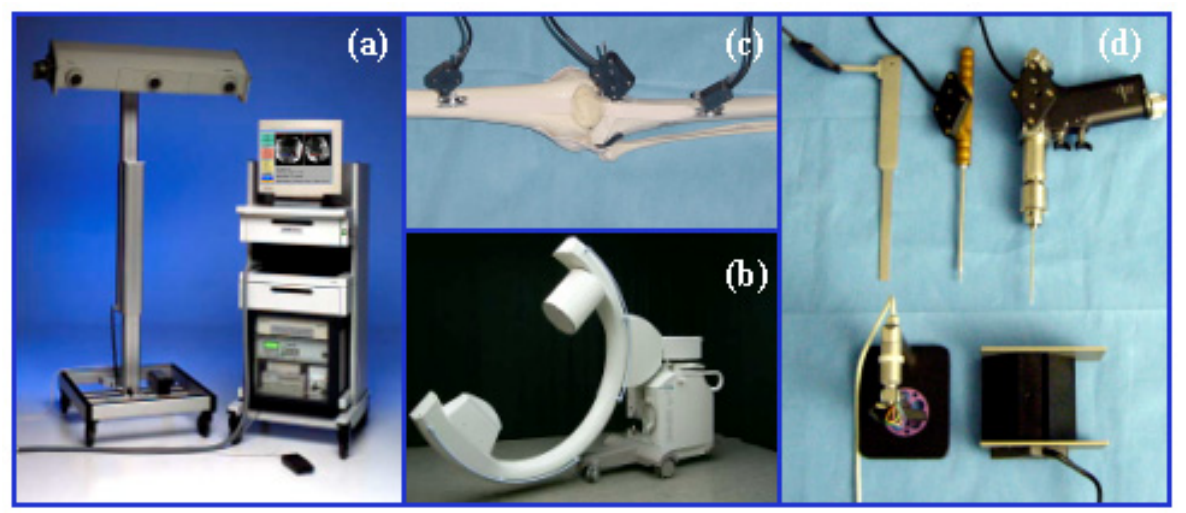

Fig. 2. System components consist of (a) optoelectric localizer and computer system, (b) registered fluoroscopic C-arm, (c) dynamical reference bases affixed to the affected limb, and (d) other navigated instruments, including navigated chisel, pointing device, navigated drill, gravitational direction measurement tool, and accuracy checker, shown in the image from top left to bottom right, respectively

Anatomic landmarks are then registered with a previous introduced hybrid concept [15], which involves kinematic pivoting movement [16], percutaneous digitization using a pointer [16], and bi-planar 3D (three-dimensional) point reconstruction based on multiple registered fluoroscopic images [17]. At the hip joint, the hip center is defined as the spherical center of the femoral head, and registered with bi-planar 3D point reconstruction using hip AP and hip lateral images. Alternatively, it can be registered through kinematic pivoting movement of the femur. At the ankle joint, the ankle center is defined as the center of the talus, and registered using ankle AP and ankle lateral images. Alternatively, it can be registered through percutaneous digitization using a palpable pointer and defined as the middle point of the transmalleolar axis formed by extreme points at lateral and medial malleoli. At the knee joint, the femoral posterior condylar axis, defined as the line between two most posterior aspects of the femoral condyles, and the tibial plateau, defined as a contour deformed with four points at the most lateral, medial, anterior and posterior edge of the tibial plateau, are registered using knee AP and knee lateral images. The knee center is defined as the geometric center of the tibial plateau.

Once the landmarks are registered, a patient specific coordinate system is established. For the mathematical description of the bone and joint geometry, each articulate joint component needs its own coordinate system. On the femoral side, the frontal plane is defined as the plane that passes through the hip and knee centers, and is parallel to the femoral posterior condyle axis; the sagittal plane passes through the hip and knee centers, and is perpendicular to the frontal plane. The transversal plane is orthogonal to both the frontal and sagittal planes. The coordinate systems of the 
proximal and distal fragments of the tibia are established with the knee, ankle centers, and affined lateral-medial direction, respectively. The affined lateral-medial direction is transferred from the femoral to the tibial side as the surgeon puts the leg into a fully extended neutral position.

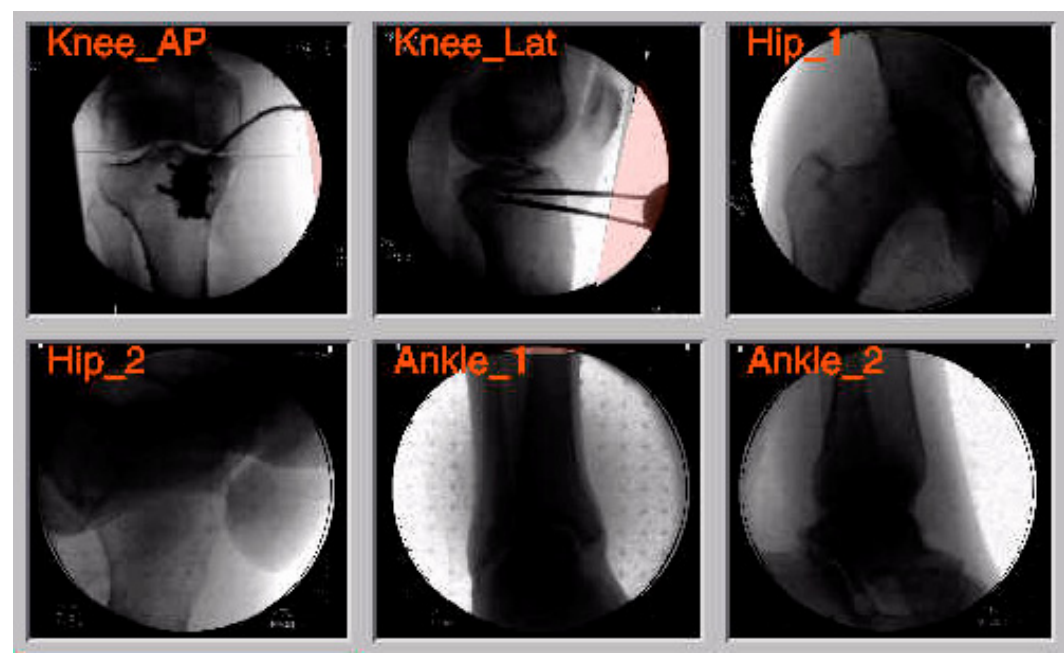

Fig. 3. Fluoroscopic images used for landmark registration and osteotomy navigation, which include knee AP, knee lateral, and optionally hip AP, hip lateral, ankle AP, and ankle lateral images

The functional parameters of the affected limb are measured intraoperatively, including varus/valgus angle, flexion/extension angle, tibial plateau slope, and joint lines orientation angle. These parameters are the basis of the surgical procedure, since only if the deformity is measured accurately, then it can be corrected correctly. We don't need any preoperative planning information derived from conventional full leg $\mathrm{X}$-ray images because it is cumbersome and error prone.

\subsection{Osteotomy Planning}

The osteotomy is planned with the aid of fluoroscopic images. The cylindrical osteotomy plane is superimposed on the fluoroscopic images in order to help the surgeon make a decision based on clinical factors including severity of the deformity, type of the fixation, soft tissues coverage, and bone quality.

With the help of the knee AP image, the surgeon determines the position of the central rotational axis, the radius of the osteotomy arc, and the distance between pinholes (Fig. 4). A numerical scalar is superimposed to help the surgeon visually control the position of the cutting plane. With the help of knee lateral image, the surgeon determines the posterior slope of the osteotomy plane. Moreover, after the surgeon specifies the anticipated postoperative alignment of the affected limb, the 
necessary rotational angle and the corresponding increment of the leg length are calculated automatically.

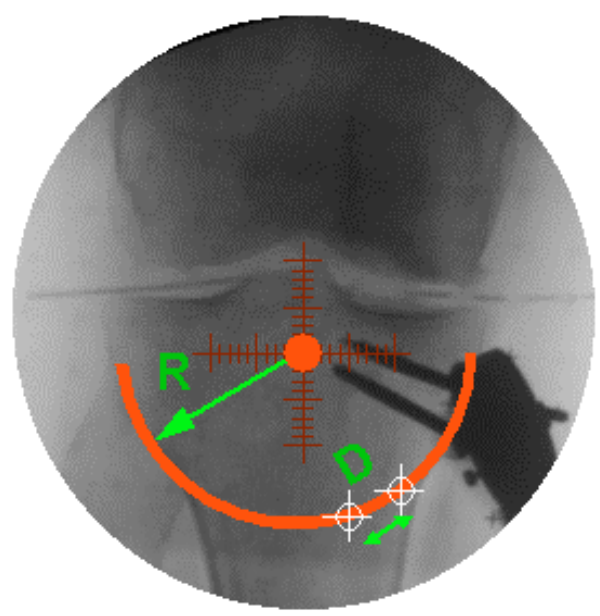

Fig. 4. Osteotomy plane is planed with the aid of fluoroscopic images. By default, the radius of the osteotomy plane $(\mathrm{R})$ is $70 \mathrm{~mm}$, the distance between rotational axis and the tibial plateau is $10 \mathrm{~mm}$, and the distance between pinholes (D) is $5 \mathrm{~mm}$. The surgeon can change these parameters according to clinical situation

\subsection{Navigational Guidance}

\section{Bone Cutting}

The accuracy of the osteotomy cannot be overemphasized. The creation of a congruous cylindrical osteotomy surface is essential in order to allow two fragments slide on each other and to provide a maximal, intimate contact between two cut surfaces, thus hastening the bone union. From the technical point of view, ideally, all drilled holes should be parallel in both the frontal and sagittal planes, and each bone cut with the osteotome should also follows the same direction. An inappropriate drilling or bone cutting, such as under-estimation of the drill depth, malorientation of the drill direction, or an excessive cut on the tibial dorsal side, may cause problems such as intercondylar fracture, pin penetration of the tibial plateau, or damage to the tibial neurovascular structures. Obviously, it is much more difficult to create such a cylindrical osteotomy surface than a straight cut.

Navigational guidance (Fig. 5) is therefore provided in order to address these technical problems. The navigated instruments used for bone cutting include a calibrated drill and a chisel. A rigid metal tube is used to prevent the bending of the drill bit during osteotomy. With virtual fluoroscopic technique, the surgeon is able to continuously monitor the position and orientation of the instruments in multiple 
fluoroscopic images simultaneously. Moreover, with real-time feedback of the deviation of the instrument to the planned cutting plane, the surgeon can minimize the risk of intraoperative technical pitfalls, and achieve a safe and accurate osteotomy.

\section{Deformity Correction}

The deformity correction is the most critical and difficult step, because once the alignment is achieved and the internal fixation implant is in place, little can be done to make further adjustments towards the ideal anticipated correction. Therefore, all the clinical relevant parameters are navigated, including three-dimensional axial alignment at the frontal, sagittal and transversal planes, the femorotibial rotational angle, the joint line orientation angle, the tibial plateau slope, and the weight-bearing axis position at the tibial plateau (Fig. 6). These parameters together can provide the surgeon with a comprehensive view of the clinical outcome, thus enabling him/her to perform the planned surgical procedure accurately.

\subsection{Surgical Procedure}

No preoperative planning is required for using the navigation system. Intraoperatively, the patient is placed on a radiolucent table as usual. Following standard exposure, two dynamical reference bases (DRB) are attached to the femur and the tibia using $2.8 \mathrm{~mm}$ Kirschner wires. After acquisition of fluoroscopic images and registration of anatomic landmarks, the deformity is measured intraoperatively and the osteotomy is planed interactively. After the surgeon is satisfied with the planning, a third DRB is affixed under navigational guidance at the proximal fragment of the tibia. The planned osteotomy and deformity correction are then performed under navigational guidance. The operation is finished in a conventional way after plate fixation.

\section{Results}

The concept of anatomic landmarks and functional parameters has been comprehensively validated in laboratory with a test bench, and the results are to be published separately [18]. It showed that the definitions of landmarks are appropriate and the algorithms for deformity measurement are accurate. With an entire leg model (RR0119, Synbone AG, Davos, Switzerland), the maximum inter-observer difference (five independent operators with identical experimental condition) was found to be $0.3^{\circ}$ for varus/valgus angle, $0.6^{\circ}$ for flexion/extension angle, and $1.0^{\circ}$ for tibial plateau slope. For the intra-observer reproducibility, 25 pairs of fluoroscopic images were acquired at different $\mathrm{C}$-arm poses (within $10^{\circ}$ deviation from the well-aligned position), and the landmarks are registered accordingly. It showed that the mean deviation was $0.4^{\circ}$ for varus/valgus angle, $0.4^{\circ}$ for flexion/extension angle, and $1.0^{\circ}$ for tibial plateau slope, with a standard deviation ( $95 \%$ confidence interval) of $0.6^{\circ}$, $0.8^{\circ}$, and $1.2^{\circ}$, respectively. 
Following the encouraging laboratory evaluation, a clinical study on focal dome osteotomy has been launched recently. Meanwhile, the study is still going on therefore no statistical results are available.
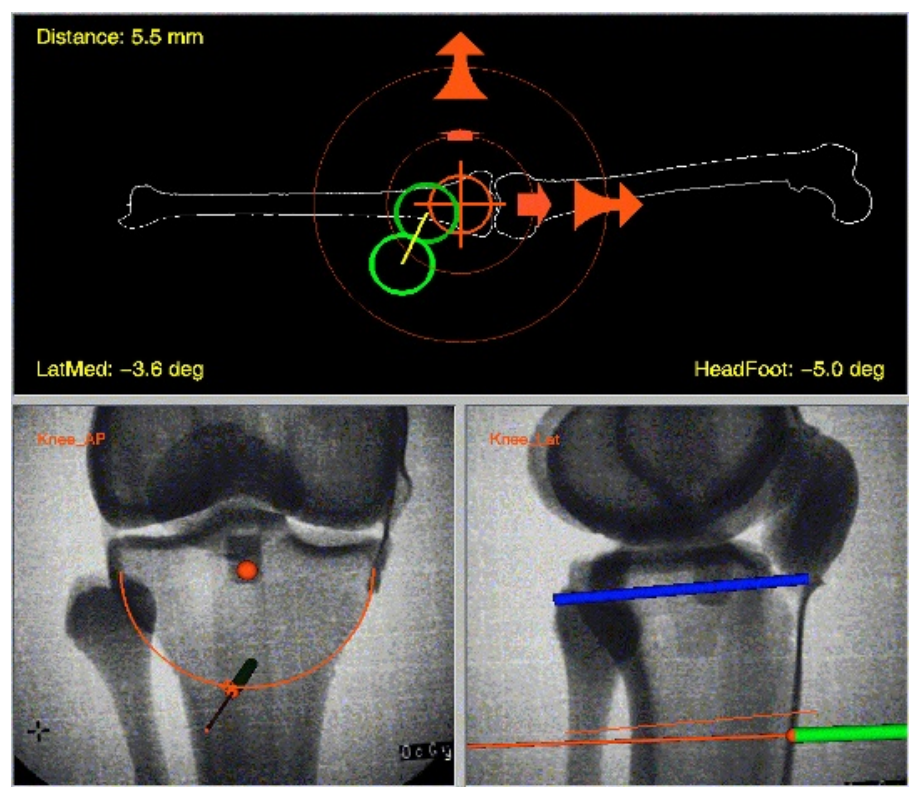

Fig. 5. The osteotomy is performed under navigational guidance. The deviation of the instrument is defined as the vector between instrument tip and current pinhole. The current active instrument, e.g., a drill, is the nearest tool to the patient. The current active pinhole, shown as the cross-circle in the figure, is the nearest pinhole on the osteotomy arc to the current active instrument. During osteotomy, the hinge axis, osteotomy arc, current pinhole, and navigated instrument are superimposed on knee AP and lateral images, in order to allow the surgeon to continually monitor the deviation and progress of the instrument. Moreover, the deviation of the instrument is intuitively displayed on the top of the window using "three circles", in which the red cross-circle is the target position, and two green circles are the tip and top of the current instrument. Four arrows are used to indicate the direction and amplitude of the further adjustment. The instrument is precisely aligned if three circles are overlapped each other

\section{Discussion}

High tibial dome osteotomy is generally recognized as complex and technical demanding. Success of such procedure depends on accurate deformity measurement, safe osteotomy with appropriate surgical technique, and precise correction of the existed deformity. 


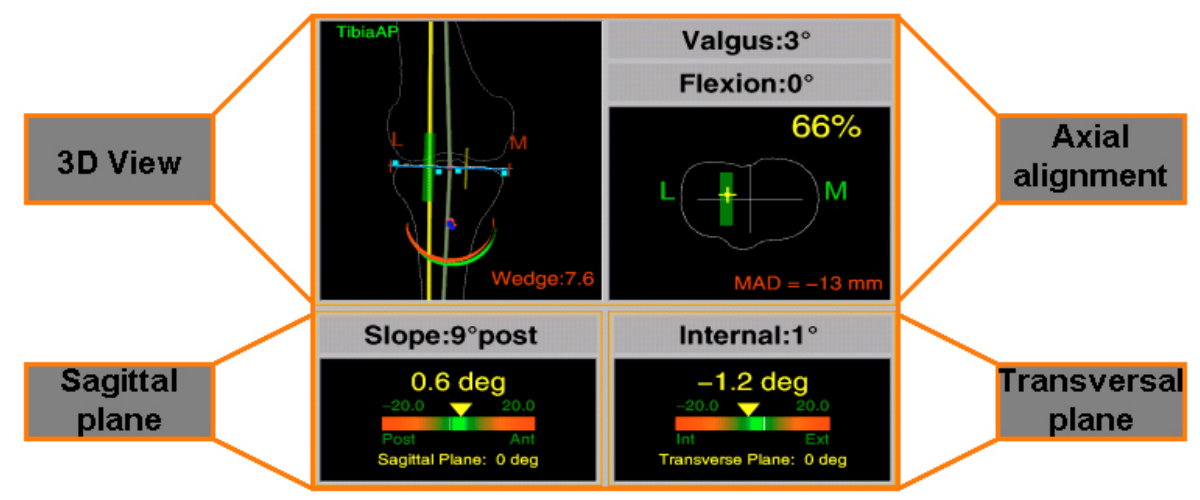

Fig. 6. The deformity is corrected under navigational guidance. The navigated parameters can be divided into three groups. The axial alignment includes varus/valgus angle ( $3^{\circ}$ valgus shown in the image), extension/flexion angle ( $0^{\circ}$ flexion), mechanical axis deviation (13 mm laterally), and the position of the weight-bearing axis at the tibial plateau $(66 \%)$. The second group includes the tibial plateau slope $\left(9^{\circ}\right.$ posterior) and the change of the slope due to osteotomy $\left(0.6^{\circ}\right.$ toward anterior). The third group consists of femorotibial rotational angle ( $1^{\circ}$ internal $)$ and the angular change between the proximal and distal fragments of the tibia $\left(1.2^{\circ}\right.$ internal $)$ in the transversal plane

Accurate registration of anatomic landmarks is the key issues of the system. A variety of methods have been adopted to help the surgeon objectively and precisely identify the landmarks on fluoroscopic images. For example, a virtual Mose circle is used for identifying the circular center of the femoral head, and a virtual ruler is used for localizing the center of the talus. In our system, the knee center is defined as the geometric center of the tibial plateau, i.e., the middle point between lateral and medial edges of the tibial plateau in the frontal plane. Our laboratory evaluation showed that these edge points are more objective and reproducible than any other anatomical features, particularly if fluoroscopic images are misaligned from true AP or lateral direction.

From the technique point of view, the work presented here only supports high tibial dome osteotomy. However, we are already in the progress of extending the current software to a more generic one, aiming to support all common osteotomy techniques around the knee joint including opening wedge, closing wedge, dome osteotomies, and to enable surgeons to perform osteotomies at either high tibial or supracondylar femoral side.

\section{Conclusions}

A CT-free computer assisted navigation system for high tibial dome osteotomy has been developed and evaluated. Although clinical study is still underway, the first trials by a group of skilled surgeons have given very positive feedbacks. This system is substantially different from other computer assisted approaches for osteotomy 
planning or robotic preparation, as it does not require any preoperative procedure or alteration of the conventional surgical procedure; neither does it need any information derived from full leg X-ray images. It allows surgeons to accurately measure the deformity, interactively plan the surgical procedure, and precisely perform the osteotomy under navigational guidance. The system thus holds the promise to reduce the risk of intraoperative complications and consequently to improve the clinical outcome of this surgical procedure.

Acknowledgement. The financial support of the AO/ASIF Foundation, Davos, Switzerland, of the M.E. Müller Foundation, Bern, Switzerland, of the Swiss National Science Foundation (NCCR/CO-ME), and of Praxim-Medivision (previously Medivision AG), Bern, Switzerland, is gratefully acknowledged.

\section{References}

1. Phillips MJ, Krackow KA: High Tibial Osteotomy and Distal Femoral Osteotomy for Valgus or Varus Deformity Around the Knee. AAOS Instructional Course Lecture, Vol.47, 429-436

2. Jackson JP, Waugh W: The Techniques and Complications of Upper Tibial Osteotomy: a Review of 226 Operations. JBJS[Br] 56-B(1974)236-45

3. Paley D: Principles of Deformity Correction. With editorial assistance from Herzenberg J. ISBN 3-540-41665-X, Springer-Verlag, Berlin Heidelbery New York (2002)

4. Hankemeire S, Paley D, Pape HC, Zeichen J, Gosling T, Kretteck C: Die Kniegelenknahe Focal-dome-Osteotomie. Orthopäde 33(2004)170-177

5. Korn, M: A New Approach to Dome High Tibial Osteotomy. The American Journal of Knee Surgery 9(1996)13-21

6. Edgar GH, Morawski DR, Santore RF: Complications of High Tibial Osteotomy. In: Knee surgery, edited by Fu FH, Harner CD, and Vince KG. ISBN 0-683-03389-1 (1996)

7. Fujisawa Y, Masuhara R, Shiohi S: The Effect of High Tibial Osteotomy on Osteoarthritis of the Knee. Orthop Clin North Am. 10(1979)585-608

8. Noyes FR, Barber-Westin SD, TE Hewett: High Tibial Osteotomy and Ligament Reconstruction for Varus Angulated Anterior Cruciate Ligament-deficient Knees. The American J. of Sport Medicine 28-3(2000)282-293

9. Aydogdu S, Sur H: High Tibial Osteotomy for Varus Deformity of More Than 20 Degrees. Rev Chir Orthop Reparatrice Mot. 84(1997)439-46

10. Takahashi T, Wada Y, Tanaka M, Iwagawa M, Ikeuchi M, Hirose D, Yamamoto H: Dome-shaped Proximal Tibial Osteotomy Using Percutaneous Drilling for Osteoarthritis of the Knee. Arch Orthop Trauma Surg. 120(2000)32-37

11. Madan S, Ranjith, Fiddian NJ: Intermediate Follow-up of High Tibial Osteotomy: a Comparison of Two Techniques. Bull Hosp Jt Dis. 61(2002-2003)11-6

12. Ellis RE, Tso CY, Rudan JF, Harrison MM: A Surgical Planning and Guidance System for High Tibial Osteotomy. Computer Aided Surgery 4(1999)264-274

13. Phillips R, Hafez M, Mohsen A, Sheman K, Hewitt J, Browbank I, Bouazza-Marouf K: Computer and Robotic Assisted Osteotomy Around the Knee. In: MMVR 2000 - 8th Annual Medicine Meets Virtual Reality Conference, Newport Beach, January 27-30, 2000. pp 265-271

14. Wang G, Zheng G, C De Simoni, Staubli A, Schmucki D, Nolte LP: A Hybrid CT-free Computer Assisted System for High Tibial Osteotomy. In: Computer Assisted Orthopaedic Surgery, edited by Langlotz F, Davies BL and Bauer A, CAOS 2003, Marbella, Spina. p 394 
15. Zheng G, Marx A, Langlotz U, Widmer K, Buttaro M, Nolte LP: A Hybrid CT-free Navigation System for Total Hip Arthroplasty. Computer Assisted Surgery 7(2002)129145

16. Kunz M, Strsuss M, Langlotz F, Deuretzbacher G, Rüther W, Nolte LP: A non-CT Based Total Knee Arthroplasty System Featuring Complete Soft-tissues Balancing. In: Medical Image Computing and Computer-Assisted Intervention, 14-17 October 2001, Utrecht, Netherlands. p 409-415

17. Hofstetter R, Slomczykowski M, Krettek C, Koppen G, Sati M, Nolte LP: Fluoroscopy as an Imaging Means for Computer Assisted Surgical Navigation. Comp. Aided Surg. 4(1999)65-76

18. Wang G, Zheng G, Keppler P, Gebhard F, Staubli A, Müller U, Schmucki D, Flütsch S, Nolte LP: Implementation, Accuracy Evaluation, and Preliminary Clinical Trial of a CTfree Navigation System for High Tibial Opening Wedge Osteotomy. Submitted to Computer Assisted Surgery 